\title{
Energy Decay of Solutions to the Wave Equations with Linear Dissipation Localized Near Infinity
}

\author{
By \\ Kiyoshi Mochizuki* and Hideo NAKazaWA**
}

\section{$\S 1$. Introduction}

Let $\Omega \subset \mathbb{R}^{N}$ be an unbounded domain with smooth boundary. We consider the mixed initial-boundary value problem

$$
\begin{cases}w_{t t}-\Delta w+b(x, t) w_{t}=0, & (x, t) \in \Omega \times(0, \infty), \\ w(x, 0)=w_{1}(x), w_{t}(x, 0)=w_{2}(x), & x \in \Omega, \\ w(x, t)=0, & (x, t) \in \partial \Omega \times(0, \infty),\end{cases}
$$

where $w_{t}=\partial w / \partial t, w_{t t}=\partial^{2} w / \partial t^{2}, \Delta$ is the $N$-dimensional Laplacian and $b(x, t)$ is a nonnegative, bounded $C^{1}$-function. Our aim is to study the energy decay of solutions when the dissipative term $b(x, t) w_{t}$ is effective only near infinity.

Let $H^{k}(\Omega), k=0,1,2$, be the usual Sobolev space with norm

$$
\|f\|_{H^{k}}=\left\{\sum_{|\alpha| \leq k} \int_{\Omega}\left|\nabla^{\alpha} f(x)\right|^{2} d x\right\}^{1 / 2}
$$

( $\alpha$ being multi-indices), and $H_{0}^{1}(\Omega)$ be the completion in $H^{1}(\Omega)$ of the set of all smooth functions with compact support in $\Omega$. We write $H^{0}(\Omega)=L^{2}(\Omega)$ and

Communicated by T. Kawai, June 16, 1997. Revised January 16, 1998 and December 22, 2000.

2000 Mathematics Subject Classification(s): 35L05, 35L20.

* Department of Mathematics, Tokyo Metropolitan University, Hachioji Tokyo 192-0397, Japan.

e-mail: mochizuk@comp.metro-u.ac.jp

*** Department of Mathematics, Tokyo Metropolitan University, Hachioji Tokyo 192-0397, Japan.

e-mail: hideo-n@comp.metro-u.ac.jp 
$\|f\|_{L^{2}}=\|f\|$. Let $E$ be the space of all pairs $f=\left\{f_{1}, f_{2}\right\}$ of functions such that

$$
\|f\|_{E}^{2}=\left\|\left\{f_{1}, f_{2}\right\}\right\|_{E}^{2}=\frac{1}{2}\left(\left\|f_{2}\right\|^{2}+\left\|\nabla f_{1}\right\|^{2}\right)<\infty .
$$

For solution $w(t)$ of (1.1), we simply write

$$
\|w(t)\|_{E}^{2}=\left\|\left\{w(t), w_{t}(t)\right\}\right\|_{E}^{2}
$$

and call it the energy of $w(t)$ at time $t$.

Now assume

$$
\left\{w_{1}, w_{2}\right\} \in\left[H^{2}(\Omega) \cap H_{0}^{1}(\Omega)\right] \times H_{0}^{1}(\Omega)
$$

Then as is well known, the initial-boundary value problem (1.1) has a global solution in the class

$$
w(\cdot, t) \in C^{0}\left([0, \infty) ; H^{2}(\Omega)\right) \cap C^{1}\left([0, \infty) ; H_{0}^{1}(\Omega)\right) \cap C^{2}\left([0, \infty) ; L^{2}(\Omega)\right) .
$$

Moreover, the energy equation

$$
\|w(t)\|_{E}^{2}+\int_{0}^{t} \int_{\Omega} b(x, \tau) w_{t}(x, \tau)^{2} d x d \tau=\|w(0)\|_{E}^{2}
$$

holds for any $t>0$.

Since $b(x, t) \geq 0$, we see from (1.4) that the energy $\|w(t)\|_{E}^{2}$ of solution is decreasing in $t>0$. Thus, a question naturally rises whether it decays or not as $t$ goes to infinity.

The decay and nondecay problems have been studied in works of Matsumura [1], Rauch-Taylor [6], Mochizuki [2], [3] and Mochizuki-Nakazawa [4]. It is proved e.g., in [4] that the energy decays if

$$
b_{0}\left\{\left(e_{n}+r+t\right) \log \left(e_{n}+r+t\right) \cdots \log ^{[n]}\left(e_{n}+r+t\right)\right\}^{-1} \leq b(x, t) \leq b_{1}
$$

in $\Omega \times[0, \infty)$ for some $b_{0}, b_{1}>0$, and it does not in general decay if

$$
0 \leq b(x) \leq b_{2}\left\{\left(e_{n}+r\right) \log \left(e_{n}+r\right) \cdots\left[\log ^{[n]}\left(e_{n}+r\right)\right]^{\gamma}\right\}^{-1}
$$

in $\Omega \times[0, \infty)$ for some $b_{2}>0$ and $\gamma>1$. Here $r=|x|$, and the positive number $e_{n}$ and the function $\log ^{[n]}(n=0,1,2, \ldots)$ are defined by

$$
\begin{gathered}
e_{0}=1, e_{1}=e, \ldots, e_{n}=e^{e_{n-1}}, \\
\log ^{[0]} a=a, \log ^{[1]} a=\log a, \ldots, \log ^{[n]} a=\log \log ^{[n-1]} a .
\end{gathered}
$$


In this paper we restrict ourselves to the energy decay problem, and obtain a similar result as above when the dissipative term $b(x, t) w_{t}$ is effective only near infinity.

We require

(A1) $N \geq 3$ and $\mathbb{R}^{N} \backslash \Omega$ is starshaped with respect to the origin $x=0$.

(A2) $b(x, t)$ is a bounded, nonnegative $C^{1}$-function, and is not increasing in $t$;

$$
b_{t}(x, t) \leq 0 \quad \text { in } \quad \Omega \times(0, \infty) .
$$

As for the function $b(x, t)$, we consider the following three conditions:

(A3) There exist $R_{0}>0$ and $n \geq 0$ such that

$$
\begin{aligned}
& b_{0}\left\{\left(e_{n}+t\right) \log \left(e_{n}+t\right) \cdots \log ^{[n]}\left(e_{n}+t\right)\right\}^{-1} \leq b(x, t) \\
& \quad \leq b_{1}\left\{\left(e_{n}+t\right) \log \left(e_{n}+t\right) \cdots \log ^{[n-1]}\left(e_{n}+t\right)\left[\log ^{[n]}\left(e_{n}+t\right)\right]^{\delta}\right\}^{-1}
\end{aligned}
$$

in $E_{\Omega}\left(R_{0}\right) \times(0, \infty)$, where $E_{\Omega}\left(R_{0}\right)=\left\{x \in \Omega ;|x|>R_{0}\right\}$, for some $b_{0}, b_{1}>0$ and $\delta \geq 0$ satisfying $1-4 b_{0} / 3<\delta<1$.

(A3)' There exist $R_{0}>0$ and $n \geq 0$ such that

$$
\begin{gathered}
b_{0}\left\{\left(e_{n}+r+t\right) \log \left(e_{n}+r+t\right) \cdots \log ^{[n]}\left(e_{n}+r+t\right)\right\}^{-1} \leq b(x, t) \\
\leq b_{1}\left\{\left(e_{n}+r+t\right) \log \left(e_{n}+r+t\right) \cdots \log ^{[n-1]}\left(e_{n}+r+t\right)\right. \\
\left.\times\left[\log ^{[n]}\left(e_{n}+r+t\right)\right]^{\delta}\right\}^{-1}
\end{gathered}
$$

in $E_{\Omega}\left(R_{0}\right) \times(0, \infty)$ for some $b_{0}, b_{1}>0$ and $\delta \geq 0$ satisfying $1-b_{0}<\delta<1$.

(A3)" There exist $R_{0}>0$ and $n \geq 0$ such that

$$
\begin{aligned}
b_{0}\left\{\left(e_{n}+r+t\right) \log \left(e_{n}+r+t\right) \cdots \log ^{[n]}\left(e_{n}+r+t\right)\right\}^{-1} \leq b(x, t) \\
\quad \leq b_{1}\left\{\left(e_{n}+r\right) \log \left(e_{n}+r\right) \cdots \log ^{[n-1]}\left(e_{n}+r\right)\left[\log ^{[n]}\left(e_{n}+r\right)\right]^{\delta}\right\}^{-1}
\end{aligned}
$$

in $E_{\Omega}\left(R_{0}\right) \times(0, \infty)$ for some $b_{0}, b_{1}>0$ and $\delta>0$ satisfying $1-b_{0} / 2<\delta<1$.

With these conditions we shall prove the following theorem.

Theorem 1. Assume (A1) and (A2).

(i) If $b(x, t)$ satisfies (A3) and $\left\{w_{1}, w_{2}\right\}$ satisfy (1.2), then the energy of the solution to (1.1) decays as $t$ goes to infinity like

$$
\|w(t)\|_{E}^{2} \leq K\left[\log ^{[n]}\left(e_{n}+t\right)\right]^{-\mu}
$$


for some $K=K\left(w_{0}, w_{1}, n\right)>0$, where $\mu$ is a positive constant satisfying

$$
\frac{1-\delta}{2}<\mu \leq 1 \quad \text { and } \quad \mu<\frac{2 b_{0}}{3} .
$$

(ii) If $b(x, t)$ satisfies $(\mathrm{A} 3)^{\prime}$, and $\left\{w_{1}, w_{2}\right\}$ satisfy (1.2) and

$$
\int_{\Omega} \log ^{[n]}\left(e_{n}+r\right)\left\{w_{2}^{2}+\left|\nabla w_{1}\right|^{2}\right\} d x<\infty,
$$

then the energy of the solution to (1.1) decays like (1.5) as $t$ goes to infinity, where $\mu$ is a positive constant satisfying

$$
\frac{1-\delta}{2}<\mu \leq 1 \text { and } \mu<\frac{b_{0}}{2} \text {. }
$$

(iii) If $b(x, t)$ satisfies (A3)", and $\left\{w_{1}, w_{2}\right\}$ satisfy the same conditions in (ii), then the energy of the solution to (1.1) decays like (1.5) as $t$ goes to infinity, where $\mu$ is a positive constant satisfying

$$
1-\delta<\mu \leq 1 \quad \text { and } \quad \mu<\frac{b_{0}}{2}
$$

Remark 1. In (A2) the condition $b_{t}(x, t) \leq 0$ is not essential for our decay estimate above. The same conclusions of Theorem 1 hold if we require that there exists a nonnegative function $\beta(t) \in L^{1}((0, t))$ such that $b_{t}(x, t) \leq$ $\beta(t)$ in $\Omega \times(0, \infty)$.

Remark 2. If $b_{0}>3 / 4$ in (A3), then we can choose $\delta=0$. So, in this case (A3) with $n=0$ is reduced to

$$
b_{0}(1+t)^{-1} \leq b(x, t) \leq b_{1} \quad \text { in } \quad E_{\Omega}\left(R_{0}\right) \times[0, \infty),
$$

and our result is expressed as

$$
\|w(t)\|_{E}^{2} \leq K(1+t)^{-\mu}
$$

for $\mu$ satisfying $1 / 2<\mu \leq 1$ and $\mu<2 b_{0} / 3$. Similarly, if $b_{0}>1$ in (A3)', then we can choose $\delta=0$. Therefore (A3)' with $n=0$ is reduced to

$$
b_{0}(1+r+t)^{-1} \leq b(x, t) \leq b_{1} \quad \text { in } \quad E_{\Omega}\left(R_{0}\right) \times[0, \infty),
$$

and (1.11) holds with $\mu$ satisfying $1 / 2<\mu \leq 1$ and $\mu<b_{0} / 2$. On the other hand, the simplest case of (A3)" is

$$
b_{0}(1+r+t)^{-1} \leq b(x, t) \leq b_{1}(1+r)^{-\delta} \quad \text { in } \quad E_{\Omega}\left(R_{0}\right) \times[0, \infty),
$$

where $\delta$ is chosen arbitrarily in $0<\delta<1$ if $b_{0}>2$, and (1.11) holds with $\mu=1$. 
Our argument is based on two weighted energy inequalities. The first one is obtained from equations (1.1) multiplied by $\{\eta w\}_{t}$ with $\eta=\varphi(t)$ or $=\varphi(r+t)$. On the other hand, the second one is obtained from equations (1.1) multiplied by $\psi\left(w_{r}+\frac{N-1}{2 r} w\right)$ with $\psi=\psi(r)$. If $b(x, t)$ is effective in the whole $\Omega$, the first weighted energy inequality is enough to obtain the energy decay. See e.g., [4]. In [4] the second inequality is used to obtain some nondecay results. In this paper we use this inequality to estimate the local energy which is not controlled by the dissipative term in which three conditions (A3), (A3)' or $(\mathrm{A} 3)^{\prime \prime}$ are required.

The energy decay for localized dissipation has been studied by Zuazua [7] and Nakao [5] when $\Omega$ is bounded. When $\Omega$ is unbounded, there are few works on this problem. Note that in Zuazua [8] is treated the Klein-Gordon equations with dissipative term:

$$
w_{t t}-\Delta w+\alpha w+f(w)+b(x) w_{t}=0, \quad \text { in } \quad \mathbb{R}^{N} \times(0, \infty),
$$

where $\alpha>0$ and $b(x) \geq b_{0}>0$ in $|x|>R_{0}$. Under suitable conditions on $f(w)$, he proved the exponential decay of energy based on a weighted energy method. However, the existence of the term $\alpha w$ is essential in his theory, and it seems difficult to apply it directly to our problem with $\alpha=0$.

The rest of the paper is organized as follows: In Section 2 we develop a semi-abstract theory for the energy decay. Sufficient conditions are given on the dissipative term (Proposition 2.1). The results are applied in Section 3 to prove Theorem 1. Finally, in Section 4 we discuss a more general equation of the form

$$
w_{t t}-\nabla \cdot\{a(x) \nabla w\}+b(x, t) w_{t}=0, \quad \text { in } \quad \Omega \times(0, \infty) .
$$

\section{§2. Weighted Energy Estimates and the Energy Decay}

Let $\eta(r, t)$ be a smooth function of $r=|x|$ and $t \geq 0$. We multiply by $\{\eta(r, t) w\}_{t}$ the both sides of (1.1). It then follows that

$$
X_{t}+\nabla \cdot Y+Z=0
$$

where

$$
\begin{aligned}
X= & \frac{1}{2} \eta\left\{w_{t}^{2}+|\nabla w|^{2}\right\}+\eta_{t} w_{t} w+\frac{1}{2}\left(\eta_{t} b-\eta_{t t}\right) w^{2}, \\
Y= & -\left(\eta w_{t}+\eta_{t} w\right) \nabla w \\
Z= & \left(\eta b-\frac{3}{2} \eta_{t}\right) w_{t}^{2}+\frac{1}{2} \eta_{t}|\nabla w|^{2}+\eta_{r} w_{r} w_{t}+\eta_{t r} w_{r} w \\
& +\frac{1}{2}\left(\eta_{t t t}-\eta_{t t} b-\eta_{t} b_{t}\right) w^{2} .
\end{aligned}
$$


We use this equation choosing $\eta=\varphi(t)$ or $\eta=\varphi(r+t)$ with $\varphi(s), s \geq 0$, satisfying

$$
\begin{gathered}
\varphi(s) \geq 1, \varphi^{\prime}(s)>0, \varphi^{\prime \prime}(s) \leq 0, \varphi^{\prime \prime \prime}(s) \geq 0 \quad \text { and } \quad \lim _{s \rightarrow \infty} \varphi(s)=\infty \\
\varphi^{\prime}(s) \varphi^{\prime \prime \prime}(s)-\varphi^{\prime \prime}(s)^{2} \geq 0
\end{gathered}
$$

In the case $\eta(r, t)=\varphi(r+t)$, we define a weighted energy of solutions as follows:

$$
\|w(t)\|_{E_{\varphi}}^{2}=\frac{1}{2} \int_{\Omega} \varphi(r+t)\left(w_{t}^{2}+|\nabla w|^{2}\right) d x
$$

and require that the initial data satisfy other than (1.2),

$$
\|w(0)\|_{E_{\varphi}}^{2}<\infty \quad(\text { cf., }(1.5))
$$

In the following we denote by $C_{i}, i=0,1,2, \ldots$, positive constants independent of $w(t)$.

Lemma 2.1. $\quad$ Let $\varphi(t)$ satisfies $(2.2),(2.3)$ and

$$
(2-\epsilon) \varphi(t) b(x, t) \geq 3 \varphi^{\prime}(t) \quad \text { in } \quad E_{\Omega}\left(R_{0}\right) \times[0, \infty)
$$

for some $0<\epsilon<1$. Then the solution $w(t)$ of (1.1) admits an inequality

$$
\begin{gathered}
\frac{1}{3} \varphi(t)\|w(t)\|_{E}^{2}-\int_{T}^{t} \int_{B_{\Omega}\left(R_{0}\right)} \frac{3}{2} \varphi^{\prime} w_{t}^{2} d x d \tau \\
+\int_{T}^{t} \int_{\Omega}\left\{\frac{\epsilon}{2} \varphi b w_{t}^{2}+\frac{1}{2} \varphi^{\prime}|\nabla w|^{2}\right\} d x d \tau \leq C_{0}(1+T)\|w(0)\|_{E}^{2}+C_{1}\left\|w_{1}\right\|^{2}
\end{gathered}
$$

for any $0 \leq T<t$, where $B_{\Omega}\left(R_{0}\right)=\left\{x \in \Omega ;|x|<R_{0}\right\}$.

Proof. We choose $\eta=\varphi(t)$ in (2.1). Let $R$ be enough large as $R>R_{0}>0$. We integrate $(2.1)$ over $B_{\Omega}(R) \times(0, t)$. Then integrations by parts give

$$
\begin{aligned}
& \int_{B_{\Omega}(R)} X(x, t) d x+\int_{0}^{t} \int_{S_{\Omega}(R)} Y(x, \tau) \cdot \frac{x}{r} d S d \tau \\
& +\int_{0}^{t} \int_{B_{\Omega}(R)} Z(x, \tau) d x d \tau=\int_{B_{\Omega}(R)} X(x, 0) d x
\end{aligned}
$$

where $S_{\Omega}(R)=\{x \in \Omega ;|x|=R\}$ and $B_{\Omega}(R)$ as above. By (2.2) and the 
Schwarz inequality, we obtain

$$
\begin{aligned}
X(x, 0) & \leq \varphi\left\{w_{2}^{2}+\left|\nabla w_{1}\right|^{2}\right\}+\frac{1}{2}\left(\varphi^{\prime 2}+\varphi^{\prime} b-\varphi^{\prime \prime}\right) w_{1}^{2} \\
& \leq \varphi\left\{w_{2}^{2}+\left|\nabla w_{1}\right|^{2}\right\}+C_{2} w_{1}^{2}, \\
X(x, t) & \geq \frac{1}{6} \varphi\left\{w_{t}^{2}+|\nabla w|^{2}\right\}-\frac{3}{4} \varphi^{-1} \varphi^{\prime 2} w^{2}+\frac{1}{2}\left(\varphi^{\prime} b-\varphi^{\prime \prime}\right) w^{2} \\
& \geq \frac{1}{6} \varphi\left\{w_{t}^{2}+|\nabla w|^{2}\right\}+\frac{1}{4} \varphi^{-1} \varphi^{\prime}\left(2 \varphi b-3 \varphi^{\prime}\right) w^{2} \\
& \geq \frac{1}{6} \varphi\left\{w_{t}^{2}+|\nabla w|^{2}\right\}-\frac{3}{4} \varphi^{-1} \varphi^{\prime 2} \chi_{R_{0}} w^{2},
\end{aligned}
$$

where $\chi_{R_{0}}$ is the characteristic function on $B_{\Omega}\left(R_{0}\right)$, and we have used (2.6) to obtain the last inequality. Similarly, since $\varphi^{\prime \prime \prime}-\varphi^{\prime \prime} b-\varphi^{\prime} b_{t} \geq 0$ by (A.2) and (2.2), we have

$$
\begin{aligned}
Z(x, t) & \geq \frac{1}{2}\left\{(2-\epsilon) \varphi b-3 \varphi^{\prime}\right\} w_{t}^{2}+\frac{1}{2}\left\{\epsilon \varphi b w_{t}^{2}+\varphi^{\prime}|\nabla w|^{2}\right\} \\
& \geq-\frac{3}{2} \varphi^{\prime} \chi_{R_{0}} w_{t}^{2}+\frac{1}{2}\left\{\epsilon \varphi b w_{t}^{2}+\varphi^{\prime}|\nabla w|^{2}\right\} .
\end{aligned}
$$

Moreover,

$$
\left|Y(x, \tau) \cdot \frac{x}{r}\right| \leq \varphi\left(w_{t}^{2}+w_{r}^{2}\right)+\frac{1}{2} \varphi^{-1} \varphi^{\prime 2} w^{2} .
$$

Since $w_{t}, w_{r}, w \in H^{1}(\Omega)$ by (1.3), we obtain

$$
\liminf _{R \rightarrow \infty}\left\{R \int_{S_{\Omega}(R)}\left(w_{t}^{2}+w_{r}^{2}+w^{2}\right) d S\right\}=0 .
$$

Therefore it follows from (2.9) and (2.10) that

$$
\liminf _{R \rightarrow \infty} \int_{0}^{t} \int_{S_{\Omega}(R)}\left|Y(x, \tau) \cdot \frac{x}{r}\right| d S d \tau=0 .
$$

Thus, using (2.6) and above estimates of $X(x, t), X(x, 0)$ and $Z(x, t)$, and letting $R \rightarrow \infty$ in (2.8), we obtain

$$
\begin{aligned}
& \frac{1}{3}\|w(t)\|_{E}^{2}-\frac{3}{4} \varphi(0)^{-1} \varphi^{\prime}(0)^{2} \int_{B_{\Omega}\left(R_{0}\right)} w^{2} d x-\int_{0}^{t} \int_{B_{\Omega}\left(R_{0}\right)} \frac{3}{2} \varphi^{\prime} w_{t}^{2} d x d \tau \\
& +\int_{T}^{t} \int_{\Omega}\left\{\frac{\epsilon}{2} \varphi b w_{t}^{2}+\frac{1}{2} \varphi^{\prime}|\nabla w|^{2}\right\} d x d \tau \leq 2 \varphi(0)\|w(0)\|_{E}^{2}+C_{3}\left\|w_{1}\right\|^{2}
\end{aligned}
$$

where we have used the fact that $\frac{\epsilon}{2} \varphi b w_{t}^{2}+\frac{1}{2} \varphi^{\prime}|\nabla w|^{2} \geq 0$ and have neglected the term

$$
\int_{0}^{T} \int_{\Omega}\left\{\frac{\epsilon}{2} \varphi b w_{t}^{2}+\frac{1}{2} \varphi^{\prime}|\nabla w|^{2}\right\} d x d \tau .
$$


In the left-hand side, the Hardy inequality gives

$$
\begin{aligned}
\int_{B_{\Omega}\left(R_{0}\right)} w^{2} d x & \leq \int_{\Omega} \frac{4 C_{4}}{(N-2) r^{2}} w^{2} d x \\
& \leq C_{4}\|\nabla w(t)\|^{2} \leq C_{4}\|w(0)\|_{E}^{2} .
\end{aligned}
$$

Moreover noting (1.4) and (2.2), we obtain

$$
\int_{0}^{T} \int_{B_{\Omega}\left(R_{0}\right)} \frac{3}{2} \varphi^{\prime} w_{t}^{2} d x d \tau \leq 3 T \max _{0 \leq t \leq T}\left[\varphi^{\prime}(t)\left\{\frac{1}{2} \int_{\Omega} w_{t}^{2} d x\right\}\right] \leq 3 \varphi^{\prime}(0) T\|w(0)\|_{E}^{2} .
$$

Applying (2.12) and (2.13) in (2.11), we conclude the assertion of the lemma.

Lemma 2.2. $\quad$ Let $\varphi(r+t)$ satisfies (2.2), (2.3) and

$$
(2-\epsilon) \varphi(r+t) b(x, t) \geq(3+k) \varphi^{\prime}(r+t) \quad \text { in } \quad E_{\Omega}\left(R_{0}\right) \times[0, \infty)
$$

for some $0<\epsilon<1$ and $k \geq 1$. Then the solution $w(t)$ of (1.1) admits an inequality

$$
\begin{aligned}
\frac{1}{2}\|w(t)\|_{E_{\varphi}}^{2} & -\int_{T}^{t} \int_{B_{\Omega}\left(R_{0}\right)} \frac{3+k}{2} \varphi^{\prime} w_{t}^{2} d x d \tau \\
& +\int_{T}^{t} \int_{\Omega}\left\{\frac{\epsilon}{2} \varphi b w_{t}^{2}+\frac{k-1}{2 k} \varphi^{\prime}|\nabla w|^{2}\right\} d x d \tau \\
& \leq 2\|w(0)\|_{E_{\varphi}}^{2}+C_{5}(1+T)\|w(0)\|_{E}^{2}+C_{6}\left\|w_{1}\right\|^{2}
\end{aligned}
$$

for any $0 \leq T<t$.

Proof. We choose $\eta=\varphi(r+t)$ in (2.1). Note that

$$
\begin{aligned}
& \frac{k}{2} \varphi^{\prime} w_{t}^{2}+\frac{1}{2 k} \varphi^{\prime}|\nabla w|^{2}+\varphi^{\prime} w_{r} w_{t}+\varphi^{\prime \prime} w_{r} w \\
& \quad=\frac{k}{2} \varphi^{\prime}\left|\frac{x}{r} w_{t}+\frac{1}{k} \nabla w+\frac{x}{r} \varphi^{\prime-1} \varphi^{\prime \prime} w\right|^{2}-\frac{k}{2} \varphi^{\prime-1} \varphi^{\prime \prime 2} w^{2}-k \varphi^{\prime \prime} w_{t} w
\end{aligned}
$$

where

$$
-k \varphi^{\prime \prime} w_{t} w=-\frac{k}{2} \partial_{t}\left[\varphi^{\prime \prime} w^{2}\right]+\frac{k}{2} \varphi^{\prime \prime \prime} w^{2} .
$$

Then by means of (A2), (2.2) and (2.3), we have

$$
\begin{aligned}
Z & \geq\left(\varphi b-\frac{k+3}{2} \varphi^{\prime}\right) w_{t}^{2}+\frac{k-1}{2 k} \varphi^{\prime}|\nabla w|^{2} \\
& +\frac{1}{2}\left\{(k+1) \varphi^{\prime \prime \prime}-k \varphi^{\prime-1} \varphi^{\prime \prime 2}\right\} w^{2}-\frac{k}{2} \partial_{t}\left[\varphi^{\prime \prime} w^{2}\right] \\
& \geq\left(\varphi b-\frac{k+3}{2} \varphi^{\prime}\right) w_{t}^{2}+\frac{k-1}{2 k} \varphi^{\prime}|\nabla w|^{2}-\frac{k}{2} \partial_{t}\left[\varphi^{\prime \prime} w^{2}\right] .
\end{aligned}
$$


Moreover, as is easily verified,

$$
\begin{aligned}
X(x, t) & -\frac{k}{2} \varphi^{\prime \prime}(r+t) w(x, t)^{2} \\
& \geq \frac{1}{4} \varphi\left\{w_{t}^{2}+|\nabla w|^{2}\right\}+\frac{1}{2} \varphi^{-1} \varphi^{\prime}\left\{\varphi b-2 \varphi^{\prime}\right\} w^{2}, \\
X(x, 0) & -\frac{k}{2} \varphi^{\prime \prime}(r) w(x, 0)^{2} \leq \varphi\left\{w_{2}^{2}+\left|\nabla w_{1}\right|^{2}\right\}+C_{7} w_{1}^{2} .
\end{aligned}
$$

We integrate (2.1) over $B_{\Omega}(R) \times(0, t)$, where $R>R_{0}$. Then the above inequalities and (2.14) give

$$
\begin{aligned}
& \frac{1}{4} \int_{B_{\Omega}(R)} \varphi\left\{w_{t}^{2}+|\nabla w|^{2}\right\} d x \\
& \quad-\varphi(0)^{-1} \varphi^{\prime}(0)^{2} \int_{B_{\Omega}\left(R_{0}\right)} w(t)^{2} d x-\int_{0}^{t} \int_{B_{\Omega}\left(R_{0}\right)} \frac{k+3}{2} \varphi^{\prime} w_{t}^{2} d x d \tau \\
& \quad+\int_{T}^{t} \int_{B_{\Omega}(R)}\left\{\frac{\epsilon}{2} \varphi b w_{t}^{2}+\frac{k-1}{2 k} \varphi^{\prime}|\nabla w|^{2}\right\} d x d \tau \\
& \leq \int_{B_{\Omega}(R)}\left\{\varphi\left(w_{2}^{2}+\left|\nabla w_{1}\right|^{2}\right)+C_{7} w_{1}^{2}\right\} d x-\int_{0}^{t} \int_{S_{\Omega}(R)} Y(x, \tau) \cdot \frac{x}{r} d S d \tau
\end{aligned}
$$

Note that

$$
\left|Y(x, \tau) \cdot \frac{x}{r}\right| \leq \varphi\left(w_{t}^{2}+w_{r}^{2}\right)+\frac{1}{2} \varphi^{-1} \varphi^{\prime 2} w^{2} .
$$

Then since $\varphi(s)=O(s)$ as $s \rightarrow \infty$, it follows that

$$
\liminf _{R \rightarrow \infty} \int_{0}^{t} \int_{S_{\Omega}(R)}\left|Y(x, \tau) \cdot \frac{x}{r}\right| d S d \tau=0 .
$$

Thus, letting $R \rightarrow \infty$ in (2.16) and taking account of (2.12) and (2.13), we conclude (2.15).

Next, let $\psi(s), s \geq 0$, be a smooth function satisfying

$$
0 \leq \psi(s) \leq \psi_{0}, \quad 0 \leq \psi^{\prime}(s) \leq s^{-1} \psi(s) \quad \text { and } \quad \psi^{\prime \prime}(s) \leq 0
$$


We multiply by $\psi(r)\left(w_{r}+\frac{N-1}{2 r} w\right)$ the both sides of equation (1.1). Then since

$$
\begin{aligned}
w_{t t} \psi\left(w_{r}+\frac{N-1}{2 r} w\right) & =\left[\psi w_{t}\left(w_{r}+\frac{N-1}{2 r} w\right)\right]_{t}-\frac{1}{2} \nabla \cdot\left[\frac{x}{r} \psi w_{t}^{2}\right]+\frac{1}{2} \psi^{\prime} w_{t}^{2}, \\
\Delta w \psi\left(w_{r}+\frac{N-1}{2 r} w\right) & =-\nabla \cdot\left(\psi \nabla w w_{r}-\frac{x}{2 r} \psi|\nabla w|^{2}\right)+\psi^{\prime} w_{r}^{2} \\
& +\frac{1}{r} \psi\left(|\nabla w|^{2}-w_{r}^{2}\right)-\frac{1}{2}\left(\frac{N-1}{r} \psi+\psi^{\prime}\right)|\nabla w|^{2} \\
& -\nabla \cdot\left\{\frac{N-1}{2 r} \psi \nabla w w+\frac{x}{2 r}\left(\frac{N-1}{2 r^{2}} \psi-\frac{N-1}{2 r} \psi^{\prime}\right) w^{2}\right\}, \\
b w_{t} \psi\left(w_{r}+\frac{N-1}{2 r} w\right) & =\psi b w_{t} w_{r}+\left(\psi b \frac{N-1}{4 r} w^{2}\right)_{t}-\psi b_{t} \frac{N-1}{4 r} w^{2},
\end{aligned}
$$

it follows that

$$
\widetilde{X}_{t}+\nabla \cdot \widetilde{Y}+\widetilde{Z}=0
$$

where

$$
\begin{aligned}
\widetilde{X}= & \psi w_{t}\left(w_{r}+\frac{N-1}{2 r} w\right)+\psi b \frac{N-1}{4 r} w^{2}, \\
\widetilde{Y}= & -\frac{x}{2 r} \psi\left\{w_{t}^{2}-|\nabla w|^{2}\right\}-\psi \nabla w\left(w_{r}+\frac{N-1}{2 r} w\right) \\
& -\frac{x}{r}\left(r^{-1} \psi-\psi^{\prime}\right) \frac{N-1}{4 r} w^{2}, \\
\widetilde{Z}= & \psi b w_{t} w_{r}+\frac{1}{2} \psi^{\prime}\left\{w_{t}^{2}+|\nabla w|^{2}\right\}-\left(\psi^{\prime \prime}+\psi b_{t}\right) \frac{N-1}{4 r} w^{2} \\
& +\left(r^{-1} \psi-\psi^{\prime}\right)\left\{|\nabla w|^{2}-w_{r}^{2}+\frac{(N-1)(N-3)}{4 r^{2}} w^{2}\right\} .
\end{aligned}
$$

Lemma 2.3. The solution $w(t)$ of (1.1) admits an inequality

$$
\begin{array}{r}
\int_{T}^{t} \int_{\Omega}\left\{\psi b w_{t} w_{r}+\frac{1}{2} \psi^{\prime}\left(w_{t}^{2}+|\nabla w|^{2}\right)\right\} d x d \tau \\
\leq C_{8}\left\{(1+T)\|w(0)\|_{E}^{2}+\left\|w_{1}\right\|^{2}\right\}
\end{array}
$$

for any $0 \leq T<t$.

Proof. Since $N \geq 3$, it follows from (A2) and (2.17) that

$$
\widetilde{Z} \geq \psi b w_{t} w_{r}+\frac{1}{2} \psi^{\prime}\left\{w_{t}^{2}+|\nabla w|^{2}\right\}
$$


Integrate (2.18) over $\Omega \times(0, t)$. Then $(2.20)$ gives

$$
\begin{aligned}
\left.\int_{\Omega} \tilde{X} d x\right|_{\tau=0} ^{\tau=t} & +\int_{0}^{t} \int_{\partial \Omega} \nu \cdot \widetilde{Y} d S d \tau \\
& +\int_{0}^{t} \int_{\Omega}\left\{\psi b w_{t} w_{r}+\frac{1}{2} \psi^{\prime}\left(w_{t}^{2}+|\nabla w|^{2}\right)\right\} d x d \tau \leq 0
\end{aligned}
$$

where $\nu$ is the outer unit normal to the bounday $\partial \Omega$. As is easily seen,

$$
\int_{\Omega} \tilde{X}(x, t) d x \geq \int_{\Omega} \psi w_{t}\left(w_{r}+\frac{N-1}{2 r} w\right) d x \geq-C_{9}\|w(0)\|_{E}^{2}
$$

for any $t \geq 0$, and

$$
\begin{gathered}
\int_{\Omega} \tilde{X}(x, 0) d x \leq C_{10}\left(\|w(0)\|_{E}^{2}+\left\|w_{1}\right\|^{2}\right) \\
\int_{0}^{T} \int_{\Omega}\left\{\psi b w_{t} w_{r}+\frac{1}{2} \psi^{\prime}\left(w_{t}^{2}+|\nabla w|^{2}\right)\right\} d x d \tau \geq-C_{11} T\|w(0)\|_{E}^{2}
\end{gathered}
$$

On the other hand, by means of the boundary condition $\left.w\right|_{\partial \Omega}=0$,

$$
\begin{aligned}
\int_{0}^{t} \int_{\partial \Omega} \nu \cdot \tilde{Y} d S d \tau & =\frac{1}{2} \int_{0}^{t} \int_{\partial \Omega} \psi\left\{\left(\nu \cdot \frac{x}{r}\right)|\nabla w|^{2}-2(\nu \cdot \nabla w)\left(\frac{x}{r} \cdot \nabla w\right)\right\} d S d \tau \\
& =-\frac{1}{2} \int_{0}^{t} \int_{\partial \Omega} \psi\left(\nu \cdot \frac{x}{r}\right)|\nu \cdot \nabla w|^{2} d S d \tau \geq 0
\end{aligned}
$$

where in the last inequality we have used the fact that $\partial \Omega$ is starshaped, i.e., $(\nu \cdot x / r) \leq 0$ on $\partial \Omega$.

Summarizing these inequalities, we conclude (2.19).

We are now ready to prove the following proposition which gives sufficient conditions on the energy decay.

Proposition 2.1. Let $w(t)$ be the solution to (1.1).

(a) Assume other than (2.6),

$$
\begin{aligned}
& \psi^{\prime}(r)-3 \varphi^{\prime}(t) \geq 0 \quad \text { in } \quad B_{\Omega}\left(R_{0}\right) \times\left[T_{0}, \infty\right), \\
& \epsilon \varphi(t) \varphi^{\prime}(t)-\psi_{0}^{2} b(x, t) \geq 0 \quad \text { in } \quad \Omega \times\left[T_{0}, \infty\right)
\end{aligned}
$$

for some $0<\epsilon<1$ and $T_{0} \geq 0$. Then we have

$$
\varphi(t)\|w(t)\|_{E}^{2} \leq C_{12}\left\{\left(1+T_{0}\right)\|w(0)\|_{E}^{2}+\left\|w_{1}\right\|^{2}\right\}<\infty .
$$


(b) Assume other than (2.14),

$$
\begin{gathered}
\psi^{\prime}(r)-(k+3) \varphi^{\prime}(r+t) \geq 0 \quad \text { in } \quad B_{\Omega}\left(R_{0}\right) \times\left[T_{0}, \infty\right), \\
\epsilon \varphi(r+t) \varphi^{\prime}(r+t)-\frac{k}{k-1} \psi_{0}^{2} b(x, t) \geq 0 \quad \text { in } \quad \Omega \times\left[T_{0}, \infty\right)
\end{gathered}
$$

for some $0<\epsilon<1, k>1$ and $T_{0} \geq 0$. Then we have

$$
\|w(t)\|_{E_{\varphi}}^{2} \leq C_{13}\left\{\|w(0)\|_{E_{\varphi}}^{2}+\left(1+T_{0}\right)\|w(0)\|_{E}^{2}+\left\|w_{1}\right\|^{2}\right\}<\infty .
$$

(c) Assume other than (2.14) and (2.24) with $k=1$,

$$
\epsilon \varphi(r+t) \psi^{\prime}(r)-\psi_{0}^{2} b(x, t) \geq 0 \quad \text { in } \quad \Omega \times\left[T_{0}, \infty\right)
$$

for some $0<\epsilon<1$ and $T_{0} \geq 0$. Then we have (2.26) with different positive constant $C_{13}$.

Proof. (a) We put together inequalities (2.7) and (2.19). Then

$$
\begin{aligned}
\frac{1}{3} \varphi(t)\|w(t)\|_{E}^{2} & +\frac{1}{2} \int_{T_{0}}^{t} \int_{B_{\Omega}\left(R_{0}\right)}\left(\psi^{\prime}-3 \varphi^{\prime}\right) w_{t}^{2} d x d \tau \\
& +\frac{1}{2} \int_{T_{0}}^{t} \int_{\Omega}\left\{\epsilon \varphi b w_{t}^{2}+\varphi^{\prime}|\nabla w|^{2}+2 \psi b w_{t} w_{r}\right\} d x d \tau \\
& \leq C_{14}\left\{\left(1+T_{0}\right)\|w(0)\|_{E}^{2}+\left\|w_{1}\right\|^{2}\right\} .
\end{aligned}
$$

By (2.21) and (2.22) we see that the second and third terms of the left-hand side is nonnegative. Thus, (2.23) is concluded.

(b) We put together inequalities (2.15) and (2.19). Then

$$
\begin{aligned}
\frac{1}{2}\|w(t)\|_{E_{\varphi}}^{2} & +\frac{1}{2} \int_{T_{0}}^{t} \int_{B_{\Omega}\left(R_{0}\right)}\left\{\psi^{\prime}-(k+3) \varphi^{\prime}\right\} w_{t}^{2} d x d \tau \\
& +\frac{1}{2} \int_{T_{0}}^{t} \int_{\Omega}\left\{\epsilon \varphi b w_{t}^{2}+\left(\frac{k-1}{k} \varphi^{\prime}+\psi^{\prime}\right)|\nabla w|^{2}+2 \psi b w_{t} w_{r}\right\} d x d \tau \\
& \leq C_{15}\left\{\|w(0)\|_{E_{\varphi}}^{2}+\left(1+T_{0}\right)\|w(0)\|_{E}^{2}+\left\|w_{1}\right\|^{2}\right\}
\end{aligned}
$$

By (2.24) and (2.25) we see that the second and third terms of the left-hand side is nonnegative. Thus, (2.26) is concluded.

(c) We choose $k=1$ in (2.28). By assumptions (2.24) with $k=1$ and (2.27) we also have the nonnegativity of the two terms of the left. Thus, (2.26) is concluded with some different constant $C_{13}$. 


\section{§3. Proof of Theorem 1}

In this section we prove Theorem 1 applying Proposition 2.1. The results (a), (b) and (c) correspond respectively to the conditions (A3), (A3)' and (A3)". We put

$$
\varphi(s)=\left[\log ^{[n]}\left(e_{n}+s\right)\right]^{\mu},
$$

where $n$ and $\mu$ are as given in Theorem 1, and put

$$
\psi(s)=\psi_{0}\left\{1-\alpha(1+s)^{-\gamma+1}\right\}
$$

when $(\mathrm{A} 3)$ or $(\mathrm{A} 3)^{\prime}$ is satisfied, and

$$
\psi(s)=\psi_{0}\left\{1-\alpha\left[\log ^{[n]}\left(e_{n}+s\right)\right]^{-\gamma+1}\right\}
$$

when (A3)" is satisfied. Here $0<\alpha<\gamma^{-1}<1$ and $\psi_{0}>0$ is determined later. In case of $(\mathrm{A} 3)^{\prime \prime}$, we further require $\gamma<\mu+\delta$ in (3.3).

First, we shall show that the above $\varphi$ satisfies (2.2) and (2.3). Differentiating (3.1), we have

$$
\begin{aligned}
\varphi^{\prime}= & \mu[n]^{\mu-1}[n-1]^{-1} \cdots[2]^{-1}[1]^{-1}[0]^{-1} \\
\varphi^{\prime \prime}= & -\mu[n]^{\mu-1}[n-1]^{-1} \cdots[2]^{-1}[1]^{-1}[0]^{-2} \\
& -\mu[n]^{\mu-1}[n-1]^{-1} \cdots[2]^{-1}[1]^{-2}[0]^{-2} \\
& \cdots \\
& -\mu[n]^{\mu-1}[n-1]^{-2} \cdots[2]^{-2}[1]^{-2}[0]^{-2} \\
& -\mu(1-\mu)[n]^{\mu-2}[n-1]^{-2} \cdots[2]^{-2}[1]^{-2}[0]^{-2}, \\
\varphi^{\prime \prime \prime}= & \left\{-2 \sum_{i=0}^{n-1}[i]^{-1} \cdots[0]^{-1}-(2-\mu)[n]^{-1} \cdots[0]^{-1}\right\} \varphi^{\prime \prime} \\
& -\mu \sum_{k=1}^{n}[k]^{-1} \cdots[0]^{-1} \sum_{i=1}^{k}[n]^{\mu-1} \cdots[i]^{-1}[i-1]^{-2} \cdots[0]^{-2},
\end{aligned}
$$

where $[k]=\log ^{[k]}\left(e_{n}+s\right)(k=0,1, \ldots, n)$. These show (2.2). Note that

$$
\begin{aligned}
\varphi^{\prime \prime \prime} \geq & \left\{-2 \sum_{i=0}^{n-1}[i]^{-1} \cdots[0]^{-1}-(2-\mu)[n]^{-1} \cdots[0]^{-1}\right\} \varphi^{\prime \prime} \\
& +\sum_{k=1}^{n}[k]^{-1} \cdots[0]^{-1} \varphi^{\prime \prime} \\
= & \left\{-2[0]^{-1}-\sum_{k=1}^{n-1}[k]^{-1} \cdots[0]^{-1}-(1-\mu)[n]^{-1} \cdots[0]^{-1}\right\} \varphi^{\prime \prime} .
\end{aligned}
$$


Then

$$
\begin{aligned}
& \frac{\varphi^{\prime \prime}}{\varphi^{\prime}}=-\sum_{k=0}^{n-1}[k]^{-1} \cdots[0]^{-1}-(1-\mu)[n]^{-1} \cdots[0]^{-1}, \\
& \frac{\varphi^{\prime \prime \prime}}{\varphi^{\prime \prime}} \leq-2[0]^{-1}-\sum_{k=1}^{n-1}[k]^{-1} \cdots[0]^{-1}-(1-\mu)[n]^{-1} \cdots[0]^{-1},
\end{aligned}
$$

and it follows that

$$
\frac{\varphi^{\prime \prime \prime}}{\varphi^{\prime \prime}}-\frac{\varphi^{\prime \prime}}{\varphi^{\prime}} \leq-[0]^{-1} \leq 0 .
$$

Thus (2.3) is proved.

Next, we shall show that the above $\psi$ satisfies (2.17). Differentiating (3.3) ((3.2) is the special case of (3.3) with $n=0)$, we have

$$
\begin{aligned}
\psi^{\prime}= & \psi_{0} \alpha(\gamma-1)[n]^{-\gamma}[n-1]^{-1} \cdots[2]^{-1}[1]^{-1}[0]^{-1} \\
\leq & \psi_{0}(1-\alpha)[0]^{-1} \leq \psi_{0}\left\{1-\alpha[n]^{-\gamma+1}\right\} s^{-1}=s^{-1} \psi(s), \\
\psi^{\prime \prime}= & -\psi_{0} \alpha(\gamma-1)[n]^{-\gamma}[n-1]^{-1} \cdots[2]^{-1}[1]^{-1}[0]^{-2} \\
& -\psi_{0} \alpha(\gamma-1)[n]^{-\gamma}[n-1]^{-1} \cdots[2]^{-1}[1]^{-2}[0]^{-2} \\
& \quad \cdots \\
& -\psi_{0} \alpha(\gamma-1)[n]^{-\gamma}[n-1]^{-2} \cdots[2]^{-2}[1]^{-2}[0]^{-2} \\
& -\psi \alpha(\gamma-1) \gamma[n]^{\gamma-1}[n-1]^{-2} \cdots[2]^{-2}[1]^{-2}[0]^{-2} \leq 0 .
\end{aligned}
$$

These show (2.17).

Now, to show Theorem 1, we have to verify that the conditions of (a), (b) or (c) in Proposition 2.1 are satisfied by the above $\varphi$ and $\psi$.

(i) First, we consider the case (A3). Note that

$$
(2-\epsilon) \varphi(t) b(x, t) \geq(2-\epsilon) b_{0} \mu^{-1} \varphi^{\prime}(t)
$$

in $E_{\Omega}\left(R_{0}\right) \times[0, \infty)$. Since $\mu<2 b_{0} / 3$, we can choose $\epsilon>0$ very small to satisfy $(2-\epsilon) b_{0} \mu^{-1}>3$. Then $(2.6)$ holds. Note that

$$
\begin{aligned}
\psi^{\prime}(r) & \geq \psi_{0} \alpha(\gamma-1)\left(1+R_{0}\right)^{-\gamma} \\
3 \varphi^{\prime}(t) & \leq 3 \mu\left\{\left(e_{n}+t\right) \cdots \log ^{[n-1]}\left(e_{n}+t\right)\left[\log ^{[n]}\left(e_{n}+t\right)\right]^{1-\mu}\right\}^{-1} .
\end{aligned}
$$

Then, if $n \geq 1$ or $\mu<1$, we can choose $\psi_{0}=1$ and $T_{0}$ sufficiently large to obtain (2.21). If $n=0$ and $\mu=1$, we also obtain (2.21) choosing $\psi_{0}$ sufficiently large. Further, note that

$$
\epsilon \varphi(t) \varphi^{\prime}(t)=\epsilon \mu\left\{\left(e_{n}+t\right) \cdots \log ^{[n-1]}\left(e_{n}+t\right)\left[\log ^{[n]}\left(e_{n}+t\right)\right]^{1-2 \mu}\right\}^{-1}
$$


and $1-2 \mu<\delta$. Then for any $\psi_{0}>0$, we can choose $T_{0}$ sufficently large to obtain (2.22). Thus, the conditions of (a) are all verified to hold.

(ii) Next we consider the case $(\mathrm{A} 3)^{\prime}$. Note that

$$
(2-\epsilon) \varphi(r+t) b(x, t) \geq(2-\epsilon) b_{0} \mu^{-1} \varphi^{\prime}(r+t)
$$

in $E_{\Omega}\left(R_{0}\right) \times[0, \infty)$. Since $\mu<b_{0} / 2$ by assumption, We can choose $\epsilon>0$ and $k>1$ to satisfy

$$
\frac{\mu}{b_{0}} \leq \frac{2-\epsilon}{k+3}<\frac{1}{2}
$$

which shows (2.14). (2.24) and (2.25) are easily shown if we follow the above argument. Thus, the conditions of (b) are all verified to hold.

(iii) Finally, we consider the case (A3)".$(2.14)$ and (2.24) with $k=1$ are already established in the above proof. Note that

$$
\begin{aligned}
\epsilon \varphi(r+t) \psi^{\prime}(r) \geq & \epsilon \psi_{0}\left(1-\gamma^{-1}\right)\left\{\left(e_{n}+r\right) \cdots \log ^{[n-1]}\left(e_{n}+r\right)\right. \\
& \left.\times\left[\log ^{[n]}\left(e_{n}+r\right)\right]^{\gamma}\left[\log ^{[n]}\left(e_{n}+r+t\right)\right]^{-\mu}\right\}^{-1}
\end{aligned}
$$

Then, since $\gamma-\delta<\mu$ by assumption, for any $\psi_{0}$, we can choose $T_{0}$ sufficiently large to obtain (2.27). Thus, the conditions of (c) are all verified to hold.

(2.22) implies (1.5) with

$$
K=C_{10}\left\{\left(1+T_{0}\right)\|w(0)\|_{E}^{2}+\left\|w_{1}\right\|^{2}\right\}
$$

and (2.25) or (2.27) implies (1.5) with

$$
K=C_{11}\left\{\|w(0)\|_{E_{\varphi}}^{2}+\left(1+T_{0}\right)\|w(0)\|_{E}^{2}+\left\|w_{1}\right\|^{2}\right\}
$$

Thus, the proof of Theorem 1 is complete.

\section{§4. A Generalization of the Problem}

In this final section, we shall remark that our results are generalized to the initial-boundary value problem

$$
\begin{cases}w_{t t}-\nabla \cdot\{a(x) \nabla w\}+b(x, t) w_{t}=0, & (x, t) \in \Omega \times(0, \infty), \\ w(x, 0)=w_{1}(x), \quad w_{t}(x, 0)=w_{2}(x), & x \in \Omega, \\ w(x, t)=0, & (x, t) \in \partial \Omega \times(0, \infty),\end{cases}
$$

with a small varying coefficient $a(x)$. For the sake of simplicity, we only consider the case where $\Omega$ and $b(x, t)$ satisfy (A1), (A2) and (A3)'. On the other hand, $a(x)$ is uniformly positive and small varying in the following sense. 
(A4) There exist $a_{0}, a_{1}>0$ such that $a_{0} \leq a(x) \leq a_{1}$ in $\Omega$. Moreover, $a(x) \in C^{1}(\Omega)$ and

$$
\frac{\beta(N-2)^{2}}{2(N-1)} \frac{\psi^{\prime}(r)}{\psi(r)} \leq \frac{a_{r}(x)}{a(x)} \leq(1-\beta) \frac{\psi^{\prime}(r)}{\psi(r)}
$$

in $\Omega$ for some $0 \leq \beta \leq 1$, where $\psi(r)$ is given in (3.2).

Multiply by $\psi(r)\left(w_{r}+\frac{N-1}{2 r} w\right)$ the both sides of (4.1). Then we have in $(2.18)$

$$
\begin{aligned}
\tilde{Z}= & \psi b w_{t} w_{r}+\frac{1}{2} \psi^{\prime} w_{t}^{2}+\frac{1}{2}\left\{\left(a \psi^{\prime}-a_{r} \psi^{\prime \prime}\right)|\nabla w|^{2}\right. \\
& \left.-a \psi^{\prime \prime} \frac{N-1}{2 r} w^{2}+a_{r}\left(\frac{\psi}{r}-\psi^{\prime}\right) \frac{N-1}{2 r} w^{2}\right\}-b_{t} \psi \frac{N-1}{4 r} w^{2} \\
& +a\left(\frac{\psi}{r}-\psi^{\prime}\right)\left\{|\nabla w|-w_{r}^{2}+\frac{(N-1)(N-3)}{2 r^{2}} w^{2}\right\} \\
\geq & \psi b w_{t} w_{r}+\frac{1}{2} \psi^{\prime} w_{t}^{2}+\frac{1}{2}\left\{\left(a \psi^{\prime}-a_{r} \psi\right)|\nabla w|^{2}\right. \\
& \left.-a \psi^{\prime \prime} \frac{N-1}{2 r} w^{2}+a_{r}\left(\frac{\psi}{r}-\psi^{\prime}\right) \frac{N-1}{2 r} w^{2}\right\} .
\end{aligned}
$$

Integrate $\tilde{Z}$ over $\Omega \times(0, t)$. Then since we have

$$
\int_{\Omega} a \psi^{\prime}|\nabla w|^{2} d x \geq \int_{\Omega}\left\{a \psi^{\prime} \frac{(N-2)^{2}}{4 r^{2}}+\left(a \psi^{\prime}\right)_{r} \frac{N-2}{2 r}\right\} w^{2} d x,
$$

it follows that

$$
\begin{aligned}
\int_{0}^{t} \int_{\Omega} \tilde{Z} d x d \tau \geq & \int_{0}^{t} \int_{\Omega}\left\{\psi b w_{t} w_{r}+\frac{1}{2} \psi^{\prime} w_{t}^{2}\right\} d x d \tau \\
& +\frac{1}{2} \int_{0}^{t} \int_{\Omega}\left\{\left((1-\beta) a \psi^{\prime}-a_{r} \psi\right)|\nabla w|^{2}\right. \\
& +\left(a \psi^{\prime} \frac{\beta(N-2)^{2}}{4 r^{2}}+a_{r} \psi \frac{N-1}{2 r^{2}}\right) w^{2} \\
& \left.+\left(-a \psi^{\prime \prime}-a_{r} \psi^{\prime}\right) \frac{N-1-\beta(N-2)}{2 r} w^{2}\right\} d x d \tau
\end{aligned}
$$

Note that $-\psi^{\prime} / \psi^{\prime \prime} \geq(\gamma-1)(1+r)^{-1} \geq \psi^{\prime} / \psi$. Then using inequalities (4.2), we obtain

$$
\int_{0}^{t} \int_{\Omega} \tilde{Z} d x d \tau \geq \int_{0}^{t} \int_{\Omega}\left\{\psi b w_{t} w_{r}+\frac{1}{2} \psi^{\prime} w_{t}^{2}\right\} d x d \tau .
$$

With this inequality we can follow the argument of Lemma 2.3 to obtain the 
Lemma 4.1. The solution $w(t)$ of (4.1) admits an inequality

$$
\int_{T}^{t} \int_{\Omega}\left\{\psi b w_{t} w_{r}+\frac{1}{2} \psi^{\prime} w_{t}^{2}\right\} d x d \tau \leq C_{16}\left\{(1+T)\|w(0)\|_{E}^{2}+\left\|w_{1}\right\|^{2}\right\}
$$

for any $0 \leq T<1$, where

$$
\|w(t)\|_{E}^{2}=\frac{1}{2} \int_{\Omega}\left\{w_{t}^{2}+a(x)|\nabla w|^{2}\right\} d x .
$$

Lemma 4.1 and a modified version of Lemma 2.2 show the following proposition which corresponds to Proposition 2.1 (b).

Proposition 4.1. Assume other than (2.14),

$$
\begin{gathered}
a(x) \psi^{\prime}(r)-(k+3) \varphi^{\prime}(r+t) \geq 0 \quad \text { in } \quad B_{\Omega}\left(R_{0}\right) \times\left[T_{0}, \infty\right), \\
\epsilon \varphi(r+t) \varphi^{\prime}(r+t)-\frac{k}{k-a_{1}} \psi_{0}^{2} b(x, t) \geq 0 \quad \text { in } \quad \Omega \times\left[T_{0}, \infty\right)
\end{gathered}
$$

for some $0<\epsilon<1, k>\max \left\{1, a_{1}\right\}$ and $T_{0} \geq 0$. Then we have

$$
\|w(t)\|_{E_{\varphi}}^{2} \leq C_{17}\left\{\|w(0)\|_{E_{\varphi}}^{2}+\left(1+T_{0}\right)\|w(0)\|_{E}^{2}+\left\|w_{1}\right\|^{2}\right\}<\infty,
$$

where

$$
\|w(t)\|_{E_{\varphi}}^{2}=\frac{1}{2} \int_{\Omega} \varphi(r+t)\left\{w_{t}^{2}+a(x)|\nabla w|^{2}\right\} d x .
$$

With this proposition we can follow the same line of proof of Theorem 1 to obtain the same conclusion for problem (4.1).

\section{Ackowledgement}

The authors wish to his sincere thanks to the referee for his careful readings.

\section{References}

[1] Matsumura, A., Energy decay of solutions of dissipative wave equations, Proc. Japan Acad., 53 (1977), 232-236.

[2] Mochizuki, K., Scattering theory for wave equations with dissipative terms, Publ. RIMS, Kyoto Univ., 12 (1976), 383-390.

[3] - Scattering Theory for Wave Equations, Kinokuniya Tokyo, 1984.

[4] Mochizuki, K. and Nakazawa, H., Energy decay and asymptotic behavior of solutions to the wave equations with linear dissipation, Publ. RIMS, Kyoto Univ., 32 (1996), 401-414. 
[5] Nakao, M., Decay of solutions of the wave equation with a local degenerate dissipation, Israel. J. Math., 95 (1996), 25-42.

[6] Rauch, J. and Taylor, M., Decaying states of perturbed wave equation, J. Math. Anal. Appl., 54 (1976), 279-285

[7] Zuazua, E., Exponential decay for the semilinear wave equation with locally distributed damping, Comm. P. D. E., 15 (1990), 205-235.

[8] - Exponential decay for the semilinear wave equations with localized damping in unbounded domains, J. Math. Pures Appl., 70 (1991), 513-529. 